\title{
The efficacy of the pocket-creation method for cases with severe fibrosis in colorectal endoscopic submucosal dissection $\square$
}

\section{다 (우우}

\section{Authors}

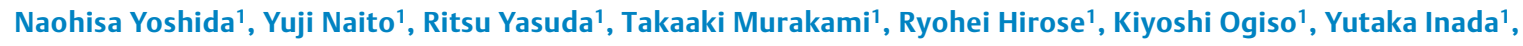
Hideyuki Konishi ${ }^{1}$, Rafiz Abdul Rani ${ }^{2}$, Mitsuo Kishimoto ${ }^{3}$, Eiichi Konishi ${ }^{3}$, Masayoshi Nakanishi ${ }^{4}$, Yoshito Itoh ${ }^{1}$

\section{Institutions}

1 Department of Molecular Gastroenterology and Hepatology, Kyoto Prefectural University of Medicine, Graduate School of Medical Science, Kyoto, Japan

2 Gastroenterology Unit, Faculty of Medicine, Universiti Teknologi MARA, Selangor, Malaysia

3 Department of Surgical Pathology, Kyoto Prefectural University of Medicine, Graduate School of Medical Science, Kyoto, Japan

4 Department of Surgery, Kyoto Prefectural University of Medicine, Graduate School of Medical Science, Kyoto, Japan

submitted 10.8.2017

accepted after revision 26.2.2018

\section{Bibliography}

DOI https://doi.org/10.1055/a-0593-5818 |

Endoscopy International Open 2018; 06: E975-E983

(c) Georg Thieme Verlag KG Stuttgart · New York

ISSN 2364-3722

\section{Corresponding author}

Naohisa Yoshida, MD, PhD, Department of Molecular Gastroenterology and Hepatology, Kyoto Prefectural University of Medicine, Graduate School of Medical Science, 465 Kajii-cho, Kawaramachi-Hirokoji, Kamigyo-ku, Kyoto 602-8566, Japan

Fax: +81-75-251-0710

naohisa@koto.kpu-m.ac.jp

\section{ABSTRACT}

Background and study aims Severe fibrosis poses a challenge in colorectal endoscopic submucosal dissection (ESD). Recently, the pocket-creation method (PCM) has been developed for overcoming various difficulties of ESD. A specific tapered hood is used for adequate traction in the
PCM, and endoscopic operability becomes stable in the pocket. In this study, we investigated the efficacy of the PCM in ESD for cases with severe fibrosis.

Patients and methods We retrospectively reviewed 1000 consecutive colorectal ESD cases (April 2006 to January 2017). Since 2016, the PCM was performed in 58 cases. The indications for ESD included (1) tumors $\geq 20 \mathrm{~mm}$ in size diagnosed as intramucosal cancer or high-grade dysplasia and part of T1a cancer using magnifying endoscopic examinations and (2) tumors that appeared impossible to resect with endoscopic mucosal resection because of suspected fibrosis. We identified 120 cases with severe fibrosis and compared them to cases without severe fibrosis. Additionally, the 120 severe fibrosis cases were divided into the PCM and non-PCM groups. En bloc resection, procedure time, discontinuation, and complications were analyzed between these 2 groups.

Results Among all 1000 ESDs, severe fibrosis and discontinuation rates were $12.0 \%$ (120 cases) and $1.8 \%$ (18 cases), respectively. Regarding the comparison between cases with severe fibrosis and with no severe fibrosis, there were significant differences about en bloc resection rate $(78.3 \%$ vs. $95.7 \%, P<0.001)$, discontinuance rate $(12.5 \%$ vs. $0.3 \%, P<$ $0.001)$, and perforation rate $(8.3 \%$ vs. $2.6 \%, P=0.001)$. Among the 120 cases with severe fibrosis, 21 and 99 cases were in the PCM and non-PCM groups, respectively. The PCM group had a higher en bloc resection rate (95.2 vs. 74.7, $P=0.03)$, a shorter mean procedure time $(\mathrm{min})(79.6$ \pm 26.5 vs. $118.8 \pm 71.0, P=0.001)$, and no cases of discontinuation. An analysis of the interobserver agreement for the diagnosis of severe fibrosis among the 3 endoscopists showed kappa values of $>0.6$.

Conclusions In cases with severe fibrosis, the PCM with ESD improved en bloc resection rates and shortened the procedure time compared to the conventional non-PCM method. Additionally, the PCM reduced the discontinuation rate. 


\section{Introduction}

The use of colorectal endoscopic submucosal dissection (ESD) is increasing worldwide [1-5]. Initially, the perforation rate was reported to be extremely high (10.4\%) [6]. Previous studies identified right-sided tumor location, tumor size $>50 \mathrm{~mm}$, severe fibrosis, poor operability, and lack of experience as risk factors for incomplete resection and perforation in colorectal ESD [5-9]. Various devices and therapeutic strategies have been developed to overcome these difficulties. Currently, several ESD knives are available for safe and precise dissection, such as the tip-shaped type, the ball-tip type, the blade type, and the scissor type [2]. Efficient submucosal elevation solutions such as hyaluronic acid are now used for higher and long-lasting elevation. The use of double-balloon endoscopy enables colorectal ESD to be performed in cases of poor operability [10].

However, despite these advancements, colorectal ESD has to be discontinued in some cases of severe fibrosis and poor operability [9]. Recently, the pocket-creation method (PCM) has been developed to overcome these difficulties in ESD $[11,12]$. A specific tapered, transparent hood is used for adequate traction in this method, which enables dissection even in cases of severe fibrosis. Additionally, endoscopic operability becomes stable in the pocket. In this study, we examined the details about cases with severe fibrosis and analyzed the efficacy of the PCM in cases of severe fibrosis compared to the conventional non-PCM method.

\section{Patients and methods}

This was a single-center retrospective study involving 1000 consecutive tumors for which ESD was performed at the Kyoto Prefectural University of Medicine from April 2006 to January 2017. The indications for ESD included (1) tumors $\geq 20 \mathrm{~mm}$ in size diagnosed as intramucosal cancer (Tis) or high-grade dysplasia (HGD) and part of T1a cancer using magnifying endoscopic examinations such as pit pattern observation, narrow band imaging, flexible spectral imaging color enhancement, and blue laser imaging and (2) tumors that appeared impossible to be resected with conventional endoscopic mucosal resection $[6,13,14]$. Thus, precheck colonoscopy before ESD was performed in all cases to identify these indications. We excluded patients who had other serious diseases or diseases requiring urgent care, such as cancer and pneumonia, and those who did not provide their consent for ESD. Six endoscopists, with experience of more than 1000 colonoscopies, performed ESD in all the patients. The PCM was adopted in our institution in March 2016 and was used for all tumors $\geq 30 \mathrm{~mm}$ in size and some of tumors $<30 \mathrm{~mm}$ in size. Regarding tumors $<30 \mathrm{~mm}$ in size, those with depression or fold convergence and recurrent tumors after endoscopic surgery were treated with the PCM because those tumors had the possibility of severe fibrosis. We reviewed medical records to collect clinicopathologic data and outcomes including age, sex, tumor size, location, morphology, severe fibrosis, antithrombotic therapy, procedure time, method (PCM or non-PCM), en bloc resection, complete resection, discontinuation rate, complications (perforation and post- operative hemorrhage), and histological diagnosis in all 1000 tumors. Then we extracted 120 tumors with severe fibrosis out of all 1000 tumors and analyzed lesion characteristics and therapeutic results of those cases compared to 880 cases with no severe fibrosis. Additionally, the 120 tumors with severe fibrosis were divided into the PCM group (21 tumors) and the nonPCM group (99 tumors) ( Fig. 1). We analyzed lesion characteristics and therapeutic results in each group. Additionally, we analyzed 880 cases with no severe fibrosis. For the analysis of the same indication between PCM and non-PCM, we excluded 343 tumors out of the 880 tumors and extracted 537 tumors with the following 3 indications: (1) tumor size $\leq 30 \mathrm{~mm}$, (2) with depression or fold convergence, and (3) recurrence after endoscopic surgery. Then we analyzed lesion characteristics and therapeutic results of the 37 tumors treated with the PCM compared to that of the 500 tumors treated with the non-PCM method.

Morphologically, polyps were divided into polypoid and nonpolypoid lesions according to the Paris endoscopic classification. Nonpolypoid lesions included elevated, flat, and depressed types. The location of the tumor was identified in accordance with the 3 segments: the right-sided colon (from the cecum to the transverse colon), the left-sided colon (from the descending to the sigmoid colon), and the rectum. Severe fibrosis was defined as a whitish muscle-like structure in the submucosal layers during ESD ( $\triangleright$ Fig. 1). There was no blue transparent layer in severe fibrosis. The evaluation of severe fibrosis was performed during and after the ESD procedure by 3 endoscopists including an operator. If the diagnosis of severe fibrosis differed among the 3 endoscopists, a consensus was reached after consultation. Additionally, we analyzed the interobserver agreement for the diagnosis of severe fibrosis among the 3 endoscopists (Endoscopist A, B, and C) using recorded movies and pictures of ESD. Additionally, intraobserver agreement was examined more than 3 wk after the initial diagnosis by each endoscopist, and it was also evaluated using recorded movies and pictures of ESD. Perforation was detected using endoscopy during ESD or using abdominal computed tomography after ESD. Postoperative hemorrhage was defined as the occurrence of hematochezia that required endoscopic treatment to stop the bleeding [6]. Histological diagnosis was performed by clinical pathologists according to the World Health Organization classification and Japanese Classification of Colorectal Carcinoma proposed by the Japanese Society for Cancer of the CoIon and Rectum $[15,16]$. Cases of mild and moderate dysplasia were diagnosed as adenomas, and those of severe dysplasia and HGD were diagnosed as Tis. T1 cancer was divided into T1a and T1b according to the submucosal invasion length (T1a: $<1000 \mu \mathrm{m}, \mathrm{T} 1 \mathrm{~b}: \geq 1000 \mu \mathrm{m})$. Complete resection was defined as cases with both histological horizontal free margins and vertical free margins. All patients provided written informed consent to undergo ESD. The ethics committees of the Kyoto Prefectural University of Medicine approved this study. This study was carried out in accordance with the Declaration of Helsinki. The obtainment of informed consent for this study participation was waived because this was a retrospective analysis of clinical practice. 


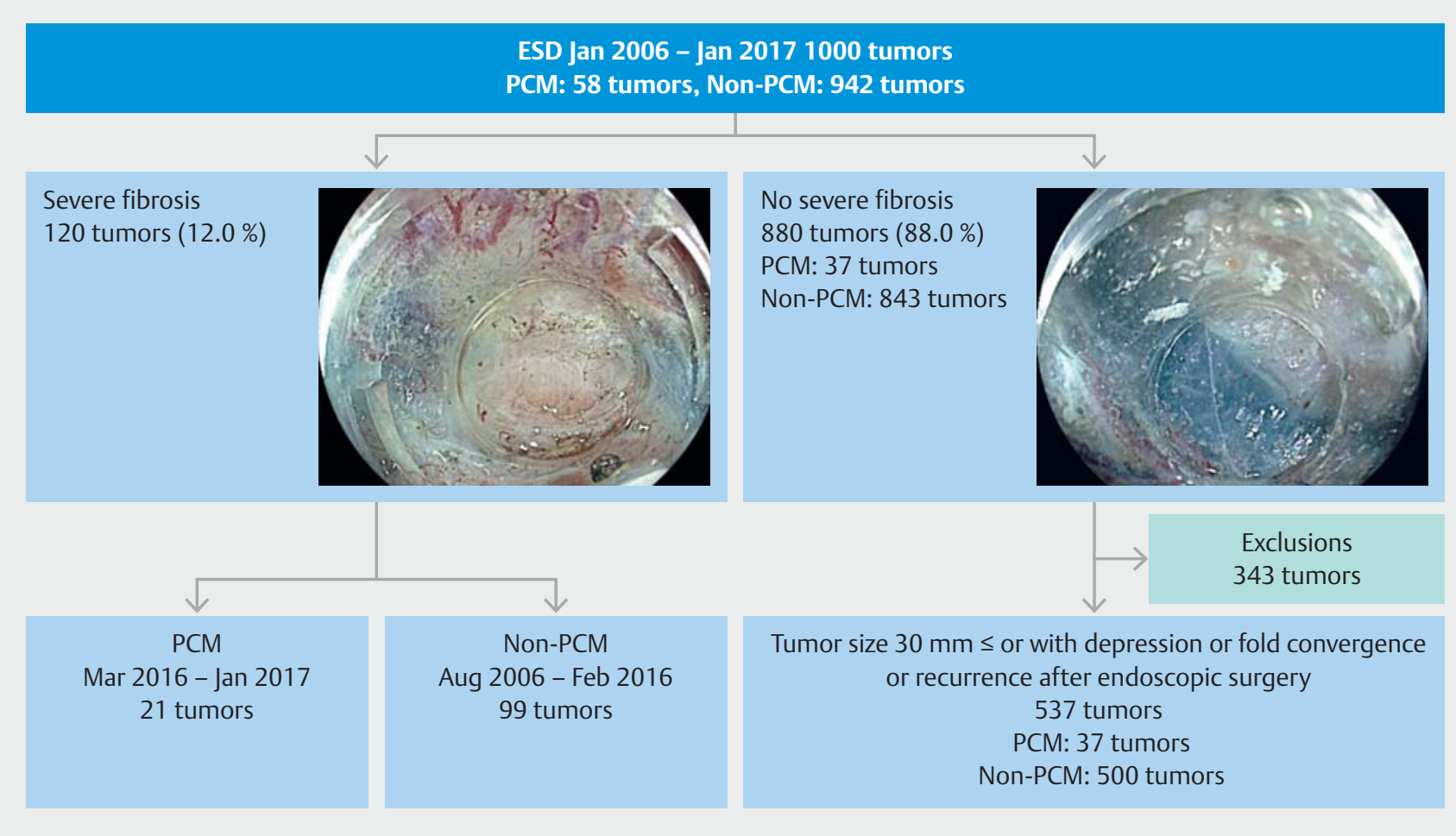

- Fig. 1 Flowchart of this study analyzing severe fibrosis in colorectal ESD.

\section{The PCM}

Our routine ESD procedure with non-PCM was described previously $[6,9]$. In brief, it was performed with a short-tipped ESD knife such as the Flush Knife BT-S $2.0 \mathrm{~mm}$ (Fujifilm Medical Co., Tokyo, Japan) and a grasping scissor knife such as the Clutch Cutter 3.5 mm (Fujifilm Medical Co.) [17, 18]. The Clutch Cutter was used only in challenging cases such as those involving several thick vessels and with poor operability. In PCM, a unique narrow transparent hood (short ST hood; Fujifilm Medical Co.) was used in addition to these 2 knives, although the ST hood was used in a previous report [11]. Initially, the anal side of the lesion was partially incised $(2-3 \mathrm{~cm})$ using the Flush Knife BT-S after submucosal injection ( $\mathbf{F i g . 2 a}$ and $\triangleright \mathbf{F i g . 2 b}$, - Video 1), followed by submucosal dissection. During the formation of the pocket, thick vessels were dissected using the Clutch Cutter, while the lesser vessels were dissected using the Flush Knife BT-S ( $\boldsymbol{F}$ Fig. $\mathbf{2 c}$ ). In the pocket, adequate traction was achieved with a stable endoscopic view, enabling safe and precise dissection of severe fibrosis ( $\triangleright$ Fig. $\mathbf{2 d}$ and $\triangleright$ Fig. 2 e, - Fig. 3). After successfully forming the pocket, a circumferential mucosal incision including an oral side incision was made ( $\triangleright$ Fig. 2f). Subsequently, submucosal dissection in the pocket was performed again until a hole (resembling a tunnel) was made in the pocket ( $\bullet$ Fig. $\mathbf{2 g}$ ). We did not make a large hole for the tunnel at the oral side to avoid decreasing the traction efficacy. Further, the remaining submucosa on both lateral sides of the pocket was dissected. Finally, the lesion was resected en bloc ( $\bullet$ Fig. $\mathbf{2 h}$ and $\triangleright$ Fig. $\mathbf{2 i}$ ).
Submucosal dissection for creating a pocket was difficult in some cases with fibrosis. In such cases, a scissor-type knife was used. Additionally, alternative traction methods such as the clip flap method were also used to create the pocket in some cases ( Fig.4a) [2]. We performed a circumferential incision when the appearance of the blue color from the submucosal injection in the oral side of a tumor was seen ( $\bullet$ Fig. $\mathbf{4 b}$ ). This finding was

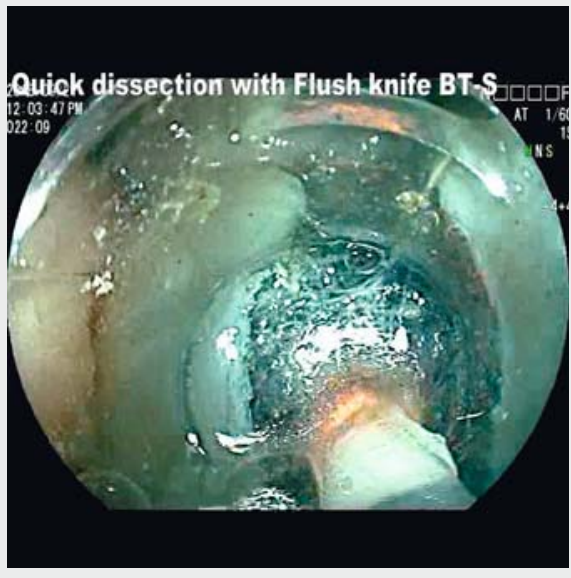

DVideo 1 Polypoid tumor $35 \mathrm{~mm}$ in size, ascending colon. PCM was performed with a Flush Knife BT-S and Clutch Cutter. Severe fibrosis was detected in the pocket. Sufficient traction was achieved, and the severe fibrosis could be dissected safely. 

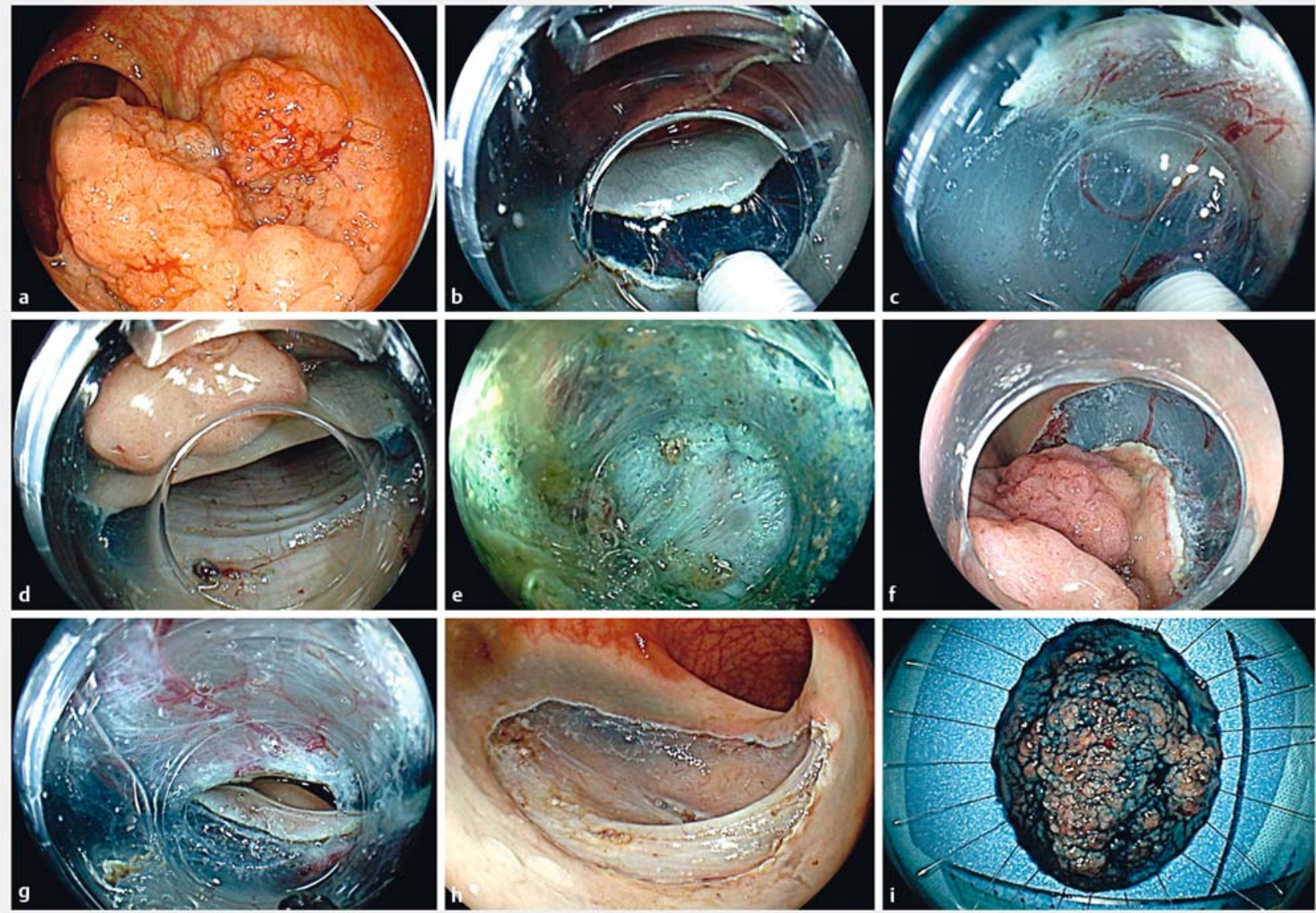

- Fig. 2 The strategy of the PCM in colorectal ESD. a A rectal tumor, $50 \mathrm{~mm}$, nonpolypoid. $\mathbf{b}$ Partial mucosal incision was performed on the anal side. c Submucosal dissection was performed to create a pocket. $\mathbf{d}$ A pocket was created. e In the pocket, adequate traction was achieved, and the endoscopic view was stable. Severe fibrosis could be dissected safely and precisely. $\mathbf{f}$ A circumferential mucosal incision was performed. $\mathbf{g}$ Dissection in the pocket was performed again followed by making a tunnel. A small hole was made to maintain the traction efficacy. h En bloc resection was achieved. The procedure time was $70 \mathrm{~min}$. i Histological diagnosis showed T1a cancer with a negative margin.
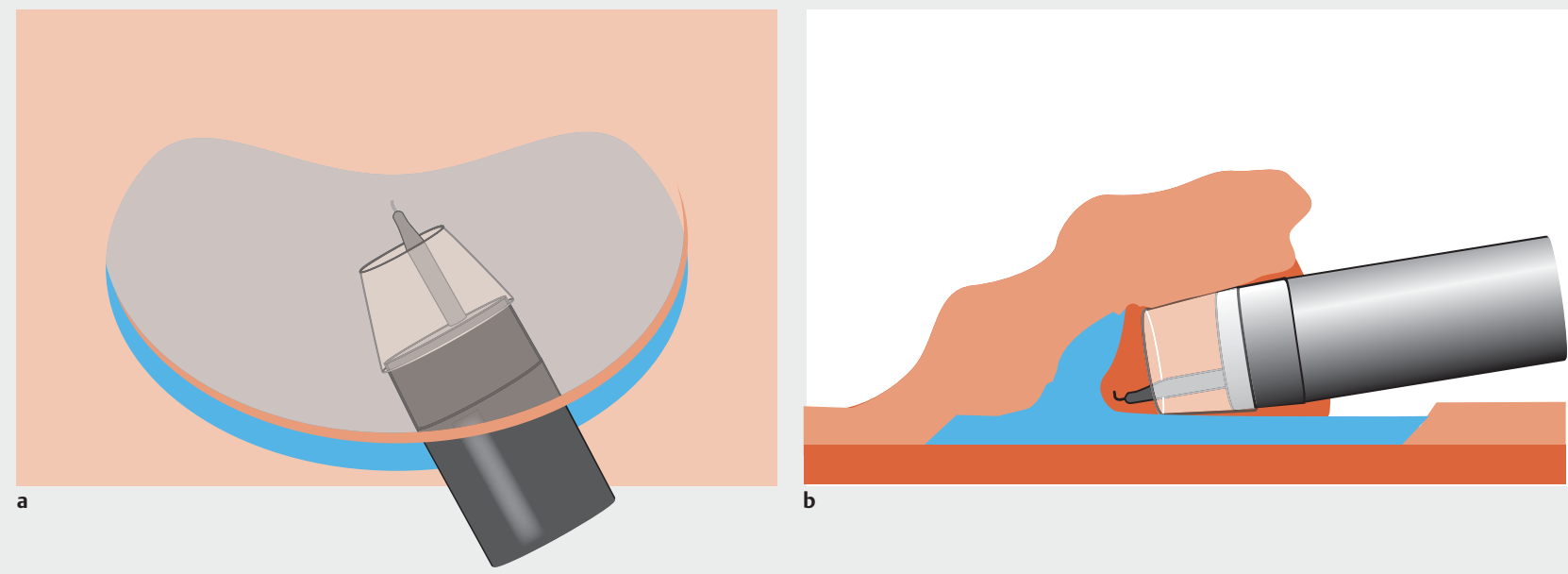

- Fig. 3 Schema of the PCM. a, b A specific tapered hood is used for adequate traction in the PCM, and endoscopic operability becomes stable in the pocket. 

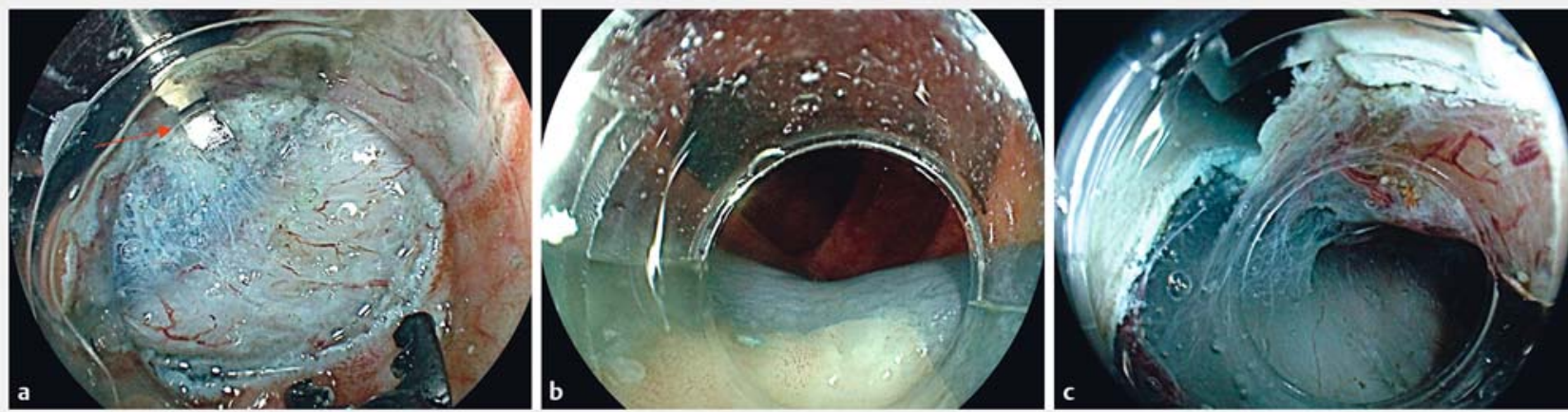

- Fig. 4 Our additional tips for successful PCM. a We used a scissor-type knife and an alternative traction method such as the clip flap method (red arrow) to create a pocket. b The timing of the circumferential incision was when the appearance of the blue submucosa was seen on the oral side of the tumor (blue color sign). $\mathbf{c}$ Both sides of the submucosa in the pocket should be thin. Thinner submucosa allows for a larger pocket, making the endoscope movement smoother.

referred to as the "blue color sign." We made both lateral sides of the submucosa thin because a thinner submucosa enabled us to create a larger pocket, facilitating easy endoscopic movement ( $\triangleright$ Fig.4c). Additionally, thinner submucosa was dissected speedy and easily.

For both PCM and non-PCM, a lower gastrointestinal endoscope with a single channel (EC-590MP, EC-L600ZP; Fujifilm Medical Co.) was used. The elevation solution was prepared with $0.4 \%$ hyaluronic acid solution (Mucoup; Boston Scientific Co., Tokyo, Japan or Seikagaku Corporation, Tokyo, Japan) and $0.2 \%$ indigo carmine (final concentration: $0.06 \%$ indigo carmine) [9]. A 25-gauge high flow needle (ImpactFlow; TOP Co., Tokyo, Japan) was used to administer the mucosal elevation solution. A high-frequency generator (VIO300D; Erbe Elektromedizin, Tubingen, Germany) and $\mathrm{CO}_{2}$ insufflation were also used. A lens cleaner was used to keep the endoscopic lens clean (Cleash; Fujifilm Medical Co., Nagase Medical Co., Tokyo, Japan) for all cases of both PCM and non-PCM since 2015 [19]. Conscious sedation was performed with midazolam and pentazocine; an automatic blood pressure monitor was used. With respect to bowel preparation, patients consumed a low-residue diet and took sodium picosulfate $10 \mathrm{~mL} 1 \mathrm{~d}$ before the examination. Since 2014, all patients also received $1.0 \mathrm{~L}$ of a highly concentrated polyethylene glycol solution with ascorbic acid (MoviPrep; Ajinomoto Pharma Co., LTD, Tokyo, Japan) in the morning on the day of the examination as described in our previous report [20]. Before then, standard PEG was used. After ESD, oral intake was resumed $2 \mathrm{~d}$ after ESD. Antibiotics were prescribed only in cases with local peritonitis and body temperature $\geq 38^{\circ} \mathrm{C}$.

\section{Statistical analysis}

Statistical analyses were performed using the Mann-Whitney $U$ test and the chi-squared test (SPSS version 22.0 for Windows; IBM Japan Ltd., Tokyo, Japan). The kappa values were calculated for inter- and intraobserver agreement for the diagnosis of severe fibrosis. A $P$ value of $<0.05$ was considered statistically significant.

\section{Results}

The therapeutic results of all 1000 ESD cases are given in $>\mathbf{T a}$ ble 1. The mean patient age was $68.1 \pm 10.1$ years, and the mean tumor size was $31.0 \pm 14.7 \mathrm{~mm}$. Severe fibrosis was seen in $12.0 \%$ of cases $(120 / 1000)$. The PCM was used in 58 cases from March 2016 to January 2017. On the other hand, 60 cases during the same period were resected with the non-PCM method, and overall, 942 cases were treated with the non-PCM method. The rates of en bloc resection and complete resection were $93.6 \%(936 / 1000)$ and $79.7 \%(797 / 1000)$, respectively. The discontinuation rate was $1.8 \%$ (18/1000). Histological diagnosis was 30 sessile serrated adenoma and polyps (SSA/P), 396 adenomas, 421 Tis/HGD, and 126 T1 cancers. The 9 cases with other histology were 4 hyperplastic polyps, 3 carcinoids, 1 mucosal prolapse syndrome, and 1 T2 cancer.

- Table 2 provides a comparison between cases with severe fibrosis and cases with no severe fibrosis. There were no significant differences in tumor size, location, or morphology, between the 2 groups. The mean procedure time ( $\mathrm{min}$ ) was significantly longer in the severe fibrosis group than in the no severe fibrosis group $(109.3 \pm 65.2$ vs. $78.0 \pm 49.1, P<0.001)$. The rate of en bloc resection in the severe fibrosis group was lower than that in the no severe fibrosis group (78.3\% vs. $95.7 \%, P<$ $0.001)$. The rate of complete resection was also significantly lower in the severe fibrosis group than in the no severe fibrosis group $(60.0 \%$ vs. $82.4 \%, P<0.001)$. The discontinuation rate was $12.5 \%$ in the severe fibrosis group and $0.3 \%$ in the no severe fibrosis group $(P<0.001)$. The 3 discontinuance cases in the no severe fibrosis group were 2 T2 cancers and 1 T3 cancer, which were diagnosed as noninvasive cancer. Regarding the reason of the discontinuance, there were muscle retractive sign due to muscle layer invasion in all 3 cases. With respect to perforation, the rate of perforation was $8.3 \%$ in the severe fibrosis group and $2.6 \%$ in the no severe fibrosis group $(P=$ $0.001)$. Histologically, the rate of T1 cancer was $25.7 \%$ in the severe fibrosis group and $26.3 \%$ in the no severe fibrosis group $(P=0.34)$. 
- Table 1 Overall therapeutic results of the 1000 colorectal ESD.

Case number

Age, mean $\pm S D$

Sex, \% (n); male/female

Tumor size, $\mathrm{mm}$, mean \pm SD (range)

Tumor location, \% (n); right-sided/left-sided/rectum

Morphology, \% (n); nonpolypoid/ polypoid

\begin{tabular}{|l|l|}
\hline Severe fibrosis, \% (n) & $12.0(120)$ \\
\hline Antithrombotic therapy, \% (n) & $11.1(111)$ \\
\hline Procedure time, min, mean \pm SD (range) & $81.5 \pm 53.2(10-420)$ \\
\hline Method, \% (n); non-PCM/PCM & $94.2(942) / 5.8(58)$ \\
\hline En bloc resection, \% (n) & $93.6(936)$ \\
\hline Complete resection, \% (n) & $79.7(797)$ \\
\hline Discontinuance case, \% (n) & $1.8(18)$ \\
\hline Perforation, \% (n) & $3.3(33)$ \\
\hline \begin{tabular}{l} 
Postoperative hemorrhage, \% (n) \\
\hline Histological diagnosis, \% (n); SSAP/Ad/ \\
Tis (HGD)/T1/others; (982 cases resect- \\
ed by ESD)
\end{tabular} & \begin{tabular}{l}
$1.8(18)$ \\
$42.9(421) / 12.8(126) /$ \\
\hline
\end{tabular}
\end{tabular}

SD: standard deviation; right-sided: cecum to transverse colon; left-sided: descending colon to sigmoid colon; SSAP: sessile serrated adenoma and polyp; Ad: adenoma
- Table 3 provides a comparison of cases with severe fibrosis between the PCM and non-PCM groups. There were no significant differences in age, sex, tumor size, morphology, or location between the 2 groups. The mean procedure time (min) was significantly shorter in the PCM group than in the nonPCM group $(79.6 \pm 26.5$ vs. $118.8 \pm 71.0, P<0.001)$. The rate of en bloc resection in the PCM group was higher than that in the non-PCM group (95.2\% vs. $74.7 \%, P=0.03)$. The rate of complete resection was significantly higher in the PCM group than in the non-PCM group ( $85.7 \%$ vs. $54.5 \%, P=0.04)$. The discontinuation rate was $0 \%$ in the PCM group and $18.2 \%$ in the nonPCM group $(P=0.05)$. With respect to complications, the rates of perforation and postoperative hemorrhage were $0 \%$ and $4.8 \%$, respectively, in the PCM group. Histologically, the rate of T1 cancer was $28.6 \%$ in the PCM group and $25.0 \%$ in the nonPCM group $(P=0.46)$. The 1 case with other histology in the $\mathrm{PCM}$ group was $\mathrm{T} 2$ cancer.

Interobserver agreement for the diagnosis of severe fibrosis was analyzed. The kappa values of interobserver agreement between the 3 endoscopists (Endoscopist A vs. Endoscopist B, Endoscopist A vs. Endoscopist C, and Endoscopist B vs. Endoscopist $C$ ) were $0.75,0.68$, and 0.76 , respectively. The kappa values of intraobserver agreement of these 3 endoscopists (A, B, and $C$ ) were $0.72,0.73$, and 0.65 , respectively.

Among the cases without severe fibrosis, the 537 cases with same indications were extracted. The cases were divided into 37 cases treated with PCM and 500 cases treated with the nonPCM method and were analyzed ( $>$ Table 4 ). There were no significant differences in tumor size $(31.1 \pm 19.3$ vs. $37.2 \pm 15.3, P$ $=0.35)$ or procedure time $(74.8 \pm 32.7$ vs. $89.6 \pm 55.4, P=0.39)$

Table 2 The comparison between cases with severe fibrosis and with no severe fibrosis.

\begin{tabular}{|c|c|c|c|}
\hline & Severe fibrosis & No severe fibrosis & \\
\hline Case number & 120 & 880 & \\
\hline Age, mean \pm SD & $66.7 \pm 10.5$ & $70.2 \pm 10.8$ & 0.17 \\
\hline Sex, \% (n); male/female & $56.7(68) / 43.3(52)$ & $56.9(501) / 43.1(379)$ & 0.95 \\
\hline Tumor size, mm, mean \pm SD (range) & $32.6 \pm 36.7(10-70)$ & $30.7 \pm 14.7(10-140)$ & 0.27 \\
\hline Tumor location, \% (n); right-sided/left-sided/rectum & $45.8(55) / 16.7(20) / 37.5(45)$ & $51.7(455) / 19.9(175) / 28.4(250)$ & 0.22 \\
\hline Morphology, \% (n); nonpolypoid/polypoid & $79.2(95) / 20.8(25)$ & $83.2(732) / 16.8(148)$ & 0.27 \\
\hline Operator, \% (n); nonexpert vs. expert & $11.7(14) / 88.3(96)$ & $43.6(384) / 56.4(496)$ & $<0.001$ \\
\hline Procedure time, min, mean \pm SD (range) & $109.3 \pm 65.2(27-320)$ & $78.0 \pm 49.1(10-420)$ & $<0.001$ \\
\hline En bloc resection, \% (n) & $78.3(94)$ & $95.7(842)$ & $<0.001$ \\
\hline Complete resection, \% (n) & $60.0(72)$ & $82.4(725)$ & $<0.001$ \\
\hline Discontinuance case, \% (n) & $12.5(15)$ & $0.3(3)$ & $<0.001$ \\
\hline Perforation, \% (n) & $8.3(10)$ & $2.6(23)$ & 0.001 \\
\hline Postoperative hemorrhage, \% (n) & $1.7(2)$ & $1.8(16)$ & 0.90 \\
\hline $\begin{array}{l}\text { Histological diagnosis, \% ( } n \text { ); SSAP/Ad/Tis (HGD)/T1/ } \\
\text { others; ( } 982 \text { cases resected by ESD) }\end{array}$ & $\begin{array}{l}4.8(5) / 34.3(36) / 34.3(36) / 25.7 \\
(27) / 0.9(1)\end{array}$ & $\begin{array}{l}2.9(25) / 41.0(360) / 43.9(385) / 26.3 \\
(99) / 0.9(8)\end{array}$ & 0.34 \\
\hline
\end{tabular}


- Table 3 The comparison of cases with severe fibrosis between the PCM and non-PCM groups.

\begin{tabular}{|c|c|c|c|}
\hline & PCM & non-PCM & $P$-value \\
\hline Case number & 21 & 99 & \\
\hline Age, mean \pm SD & $66.7 \pm 10.5$ & $70.2 \pm 10.8$ & 0.17 \\
\hline Sex, \% (n); male/female & $57.1(12) / 42.9(9)$ & $53.5(53) / 46.5(46)$ & 0.76 \\
\hline Tumor size, mm, mean \pm SD (range) & $30.1 \pm 9.5(18-50)$ & $34.5 \pm 16.5(10-70)$ & 0.23 \\
\hline Tumor location, \% (n); right-sided/left-sided/rectum & $49.7(12) / 0(0) / 29.6(9)$ & $43.4(43) / 20.2(20) / 36.4(36)$ & 0.57 \\
\hline Morphology, \% (n); nonpolypoid/polypoid & $71.4(15) / 28.6(6)$ & $73.7(73) / 25.3(26)$ & 0.67 \\
\hline Operator, \% (n); nonexpert vs. expert & $28.6(6) / 71.4(15)$ & $19.2(19) / 80.8(80)$ & 0.33 \\
\hline Procedure time, min, mean \pm SD (range) & $79.6 \pm 26.5(41-140)$ & $118.8 \pm 71.0(27-320)$ & $<0.001$ \\
\hline En bloc resection, \% (n) & $95.2(20)$ & $74.7(74)$ & 0.03 \\
\hline Complete resection, \% (n) & $85.7(18)$ & $54.5(54)$ & 0.04 \\
\hline Discontinuance case, \% (n) & $0(0)$ & $18.2(15)$ & 0.05 \\
\hline Perforation, \% (n) & $0(0)$ & $10.1(10)$ & 0.12 \\
\hline Postoperative hemorrhage, \% (n) & $4.8(1)$ & $1.0(1)$ & 0.22 \\
\hline $\begin{array}{l}\text { Histological diagnosis, \% ( } n \text { ); SSAP/Ad/Tis (HGD)/T1/ } \\
\text { other; (only lesions resected by ESD) }\end{array}$ & $\begin{array}{l}14.3(3) / 33.3(7) / 19.0(4) / 28.6(6) / \\
4.8(1)\end{array}$ & $\begin{array}{l}2.4(2) / 34.5(29) / 38.1(32) / \\
25.0(21) / 0(0)\end{array}$ & 0.46 \\
\hline
\end{tabular}

Table 4 The comparison of cases without severe fibrosis between the PCM and non-PCM groups.

\begin{tabular}{|c|c|c|c|}
\hline & PCM & non-PCM & $P$-value \\
\hline Case number & 37 & 500 & \\
\hline Age, mean \pm SD & $65.2 \pm 13.5$ & $67.6 \pm 10.6$ & 0.17 \\
\hline Sex, \% (n); male/female & $48.6(18) / 51.4(19)$ & $56.4(282) / 43.6(218)$ & 0.35 \\
\hline Tumor size, mm, mean \pm SD (range) & $31.1 \pm 19.3(10-60)$ & $37.3 \pm 15.3(10-140)$ & 0.35 \\
\hline Tumor location, \% (n); right-sided/left-sided/rectum & $43.2(16) / 21.6(8) / 35.1(13)$ & $54.0(270) / 17.0(85) / 28.8(144)$ & 0.20 \\
\hline Morphology, \% (n); nonpolypoid/polypoid & $83.8(31) / 16.2(6)$ & $76.0(430) / 14.0(70)$ & 0.71 \\
\hline Operator, \% (n); nonexpert vs. expert & $51.4(19) / 48.6 .4(18)$ & $34.2(171) / 65.8(329)$ & 0.03 \\
\hline Procedure time, min, mean \pm SD (range) & $74.8 \pm 32.7(24-170)$ & $89.6 \pm 55.4(10-420)$ & 0.39 \\
\hline En bloc resection, \% (n) & $100.0(37)$ & $94.4(472)$ & 0.03 \\
\hline Complete resection, \% (n) & $100.0(37)$ & $75.8(379)$ & $<0.001$ \\
\hline Discontinuance case, \% (n) & $0(0)$ & $0.6(3)$ & 0.43 \\
\hline Perforation, \% (n) & $0(0)$ & $2.8(14)$ & 0.18 \\
\hline Postoperative hemorrhage, \% ( $\mathrm{n}$ ) & $0(0)$ & $2.2(11)$ & 0.26 \\
\hline $\begin{array}{l}\text { Histological diagnosis, \% ( } n \text { ); SSAP/Ad/Tis (HGD)/T1/ } \\
\text { others; (only lesions resected by ESD) }\end{array}$ & $\begin{array}{l}8.1(3) / 29.7(11) / 54.1(20) / 5.4(2) / \\
2.7(1)\end{array}$ & $\begin{array}{l}1.6(8) / 35.8(179) / 50.2(251) / \\
11.4(57) / 0.4(2)\end{array}$ & 0.79 \\
\hline
\end{tabular}


between the PCM and non-PCM groups. The rate of the en bloc resection in the PCM group was significantly higher than that in the non-PCM group (100\% vs. $94.4 \%, P=0.03$ ). Additionally, the rate of the complete resection in the PCM group was significantly higher than that in the non-PCM group (100\% vs. $75.8 \%$, $P<0.001)$.

\section{Discussion}

In this study, we evaluated the efficacy of the PCM in colorectal ESD in terms of severe fibrosis. The rates of severe fibrosis and discontinuation were $12.0 \%$ and $1.8 \%$, respectively. Severe fibrosis cases were significantly related with longer procedure time, lower en bloc resection and complete resection rates, and higher discontinuance and perforation rates. Among severe fibrosis cases, the PCM group had a significantly better en bloc resection rate, complete resection rate, and mean procedure time compared to the non-PCM group. Thus, the results show that PCM effectively overcomes the difficulties associated with severe fibrosis in colorectal ESD.

Difficulties in ESD result in incomplete en bloc resection and perforation. The presence of fibrosis is related to perforation, and severe fibrosis is related to incomplete resection $[8,21]$. Our previous study showed that severe fibrosis was the most important risk factor for incomplete resection [9]. A recent report showed that submucosal invasion and carcinomatous histology were independent risk factors for severe fibrosis [22]. In our study, the en bloc resection rate in cases with severe fibrosis was $95.2 \%$ in the PCM group. Additionally, there were no cases of discontinuation because of severe fibrosis in the PCM group. Because the endoscopic view was stable in the pocket, severe fibrosis could be dissected safely and accurately. On the other hand, tumor size $\geq 50 \mathrm{~mm}$ has also been reported to be associated with incomplete resection and complications [5, 8]. This could be attributed to the fact that a partially resected specimen such as a flap during ESD hinders the subsequent procedure. In the PCM, a partially resected specimen does not form a flap, and thus there is no hindrance to the subsequent procedure. We believe the PCM is useful not only for cases of severe fibrosis but also for large tumors $\geq 50 \mathrm{~mm}$. Indeed, a report from Turkey described the efficacy of a tunneling method for resecting colorectal large tumors, which was similar to the PCM [23].

Regarding the technical aspects of the PCM, thick vessels in the pocket were detected in some cases. Those vessels were dissected carefully using a Flush Knife BT-S or Clutch Cutter according to their diameter and pulsation, as a massive hemorrhage might disturb the endoscopic view in the pocket. When a massive hemorrhage occurred, the point of hemorrhage was sometimes difficult to identify in a pocket. In those cases, we opened the pocket and found the point of hemorrhage. Additionally, the timing of circumferential incision was important. When the circumferential incision was performed early, submucosal elevation by injection liquid went down easily, and it made further dissection of severe fibrosis difficult. Thus, we performed circumferential incision when the blue color sign was detected on the oral side of a tumor.
One weakness of the PCM is scope insertion. The hood for the PCM is long and narrow, which could make insertion difficult especially in cases with severe adhesions. However, we did not encounter any such cases in which we could not insert the colonoscope to the lesion. Another weak point is the cloud of the endoscopic hood, which makes the endoscopic lens cloudy. We used a novel endoscopic lens cleaner, Cleash, to clean the lens and hood. It is primarily composed of 2 harmless nonionic surfactants, and its efficacy in preventing lens cloudiness in colorectal ESD has been reported [19]. On the other hand, our results showed that the PCM did not improve either procedure time or en bloc resection rate for cases without severe fibrosis compared to the non-PCM method. One possible reason is that the procedure speed of the PCM becomes slower with mucosal incisions because of the narrow view through the long-tapered hood compared to the non-PCM method. The other possible reason is that the non-PCM method may be developed sufficiently to achieve en bloc resection with ESD in cases without severe fibrosis.

Recently, various traction methods have been introduced to improve ESD procedures, such as the clip, clip with line, external forceps, clip and snare, and double scope methods [24]. We used some of these until we adopted the PCM in 2016. The PCM is a type of traction method that uses a narrow and long hood. Compared to other traction methods, the PCM can be applied not only in cases of severe fibrosis but also in cases of poor operability $[11,12]$. Our data show that the PCM significantly improved the therapeutic results such as procedure time, en bloc resection rate, and complete resection rate compared to the non-PCM method.

A limitation of this study was its single-center retrospective design and the small sample size of the PCM group. The definition of severe fibrosis was subjective, although the kappa values of interobserver agreement and intraobserver agreement showed good results. There was the possibility of selection bias for the PCM among cases $<30 \mathrm{~mm}$ in size because endoscopic findings such as depression and fold convergence were subjective. However, to decrease selection bias, we analyzed only cases with severe fibrosis that were detected during dissection. In all cases using the non-PCM method, there was a learning curve because they included the ESD cases in an initial period. We did not examine the factor of poor scope operability, which was reported as a risk factor for difficulties with ESD. We also did not analyze the histological evidence for severe fibrosis in cases with severe fibrosis.

In conclusion, the PCM is effective in overcoming the difficulties associated with severe fibrosis compared to non-PCM strategies. Since this study was a retrospective observational study, the result should be verified in a prospective randomized trial.

\section{Acknowledgements}

We thank all members of the Department of Molecular Gastroenterology and Hepatology, Kyoto Prefectural University of Medicine, for their help with this study. 


\section{Competing interests}

Yoshito Itoh is affiliated with a donation-funded department from Fujifilm Medical Co. Ltd. Naohisa Yoshida received a research grant from Fujifilm Medical Co. Ltd. The other authors have no conflicts of interest to declare.

\section{References}

[1] Yamamoto H, Kawata $\mathrm{H}$, Sunada K et al. Successful en-bloc resection of large superficial tumors in the stomach and colon using sodium hyaluronate and small-caliber-tip transparent hood. Endoscopy 2003; 35: $690-694$

[2] Yamamoto K, Michida T, Nishida T et al. Colorectal endoscopic submucosal dissection: recent technical advances for safe and successful procedures. World J Gastrointest Endosc 2015; 7: 1114-1128

[3] Tanaka S, Oka S, Kaneko I et al. Endoscopic submucosal dissection for colorectal neoplasia: possibility of standardization. Gastrointest Endosc 2007; 66: $100-107$

[4] Yoshizaki T, Toyonaga T, Tanaka S et al. Feasibility and safety of endoscopic submucosal dissection for lesions involving the ileocecal valve. Endoscopy 2016; 48: 639-645

[5] Saito Y, Uraoka T, Yamaguchi Y et al. A prospective, multicenter study of 1111 colorectal endoscopic submucosal dissections (with video). Gastrointest Endosc 2010; 72: 1217-1225

[6] Yoshida N, Wakabayashi N, Kanemasa K et al. Endoscopic submucosal dissection for colorectal tumors: technical difficulties and rate of perforation. Endoscopy 2009; 41: 758 - 761

[7] Kim ES, Cho KB, Park KS et al. Factors predictive of perforation during endoscopic submucosal dissection for the treatment of colorectal tumors. Endoscopy 2011; 43: 573-578

[8] Hayashi N, Tanaka S, Nishiyama S et al. Predictors of incomplete resection and perforation associated with endoscopic submucosal dissection for colorectal tumors. Gastrointest Endosc 2014; 79: 427 435

[9] Yoshida N, Fernandopulle N, Murakami T et al. Difficult manipulation is one of the risk factors for incomplete resection in colorectal endoscopic submucosal dissection. J Gastroenterol Hepatol Res 2016; 5: $2063-2069$

[10] Hotta K, Katsuki S, Ohata K et al. Efficacy and safety of endoscopic interventions using the short double-balloon endoscope in patients after incomplete colonoscopy. Dig Endosc 2015; 27: 95 - 98
[11] Hayashi Y, Miura Y, Yamamoto H. Pocket-creation method for the safe, reliable, and efficient endoscopic submucosal dissection of colorectal lateral spreading tumors. Dig Endosc 2015; 27: 534- 535

[12] Sakamoto H, Hayashi Y, Miura Y et al. Pocket-creation method facilitates endoscopic submucosal dissection of colorectal laterally spreading tumors, non-granular type. Endosc Int Open 2017; 5: E123-E129

[13] Sano Y, Ikematsu H, Fu KI et al. Meshed capillary vessels by use of narrow-band imaging for differential diagnosis of small colorectal polyps. Gastrointest Endosc 2009; 69: 278-283

[14] Kudo S, Hirota S, Nakajima T et al. Colorectal tumours and pit pattern. I Clin Pathol 1994; 47: 880-885

[15] Japanese Society for Cancer of the Colon and Rectum. Japanese Classification of Colorectal Carcinoma. 2nd ed. Tokyo, Japan: Kanehara; 2009

[16] Hamilton SR, Aaltonen LA , eds. World Health Organization Classification of Tumors. Pathology and Genetics of Tumours of the Digestive System Lyon, France: IARC Press; 2010: 104 - 109

[17] Yoshida N, Toyonaga T, Murakami T et al. Efficacy of a novel narrow knife with water jet function for colorectal endoscopic submucosal dissection. Gastro Res Pract 2017; 2017: 5897369

[18] Akahoshi K, Honda K, Akahane $\mathrm{H}$ et al. Endoscopic submucosal dissection by using a grasping-type scissors forceps: a preliminary clinical study (with video). Gastrointest Endosc 2008; 67: 1128-1133

[19] Yoshida N, Naito Y, Hirose R et al. Risk of lens cloudiness during colorectal endoscopic submucosal dissection and ability of a novel lens cleaner to maintain and restore endoscopic view. Dig Endosc 2015; 27: $609-617$

[20] Yoshida N, Naito Y, Murakami T et al. Safety and efficacy of a sameday low-volume $1 \mathrm{~L}$ PEG bowel preparation in colonoscopy for the elderly people and people with renal dysfunction. Dig Dis Sci 2016; 61: $3229-3235$

[21] Isomoto $\mathrm{H}$, Nishiyama $\mathrm{H}$, Yamaguchi $\mathrm{N}$ et al. Clinicopathological factors associated with clinical outcomes of endoscopic submucosal dissection for colorectal epithelial neoplasms. Endoscopy 2009; 41: $679-683$

[22] Kim EK, Han DS, Ro Y et al. The submucosal fibrosis: what does it mean for colorectal endoscopic submucosal dissection? Intest Res 2016; 14: $358-364$

[23] Aslan F, Akpinar Z, Yurtlu DA et al. Single tunneling method with endoscopic submucosal dissection for treatment of a rectal giant (18cm) laterally spreading tumor. Endoscopy 2017; 49: E114-E116

[24] Tsuji K, Yoshida N, Nakanishi H et al. Recent traction methods for endoscopic submucosal dissection. World J Gastroenterol 2016; 22 : $5917-5926$ 